\title{
Telephone-Delivered Dietary Intervention in Patients with Age-Related Macular Degeneration: 3-Months Post- Intervention Findings of a Randomised Controlled Trial
}

\section{Diana Tang ( $\square$ diana.tang@sydney.edu.au )}

Westmead Institute for Medical Research https://orcid.org/0000-0003-2007-9054

\section{Paul Mitchell}

Westmead Institute for Medical Research; University of Sydney

\section{George Burlutsky}

Westmead Institute for Medical Research

\section{Gerald Liew}

Westmead Institute for Medical Research; University of Sydney

\section{Victoria M. Flood}

The University of Sydney; Western Sydney Local Health District - Westmead Hospital

\section{Bamini Gopinath}

Westmead Institute for Medical Research; University of Sydney

\section{Research}

Keywords: age-related macular degeneration, education, nutrition, telehealth

Posted Date: August 28th, 2020

DOI: https://doi.org/10.21203/rs.3.rs-66118/v1

License: (c) (P) This work is licensed under a Creative Commons Attribution 4.0 International License. Read Full License 


\section{Abstract}

Background: There is an evidence-practice gap between the dietary recommendations for age-related macular degeneration (AMD) presented in the literature and those practiced by patients. This study reports on the 3months post-intervention results of a randomised controlled trial (RCT) evaluating telephone-delivered dietary counselling aiming to improve dietary behaviors among patients with AMD.

Methods: 155 AMD patients (57\% female, aged $78 \pm 8$ years; control: 78, intervention: 77 ), primarily residing in New South Wales, Australia were recruited into the RCT at baseline. Participants completed a baseline questionnaire and short dietary questionnaire for age-related macular degeneration (SDQ-AMD). The intervention included an evidence-based nutrition resource and four monthly calls with a dietitian in addition to usual care; the control arm only received usual care. Immediately post-intervention, participants in the intervention arm were invited to repeat the SDQ-AMD and complete a feedback form. At 3-months post-intervention, all participants were invited to repeat the SDQ-AMD. Statistical analyses included t-tests and McNemar's test to determine change in dietary intakes and proportion of participants meeting the dietary recommendations, respectively; level of significance for all statistical analyses was $p<0.05$. Descriptive statistics were used to describe adherence to and acceptability of the intervention.

Results: At 3-months post-intervention compared to baseline, there were significant improvements within the intervention arm including increased intakes (mean serves \pm SE) of dark green leafy vegetables ( $0.99 \pm 0.17$ vs $1.71 \pm 0.22 ; p=0.003)$ and legumes $(0.69 \pm 0.10$ vs $1.12 \pm 0.16 ; p=0.02)$, and reduced intakes of specified discretionary foods $(8.31 \pm 0.76$ vs $6.54 \pm 0.58, p=0.01)$. Between study arms, intakes of nuts were significantly higher in the intervention arm (control: $2.71 \pm 0.32$ vs intervention: $3.96 \pm 0.51 ; p=0.04$ ) at 3-months postintervention. Overall feedback about the intervention was positive, with all completers $(n=74)$ finding the program worthwhile and $98.7 \%(n=73)$ indicating they would recommend it to others.

Conclusion: This novel intervention was well-received and significantly improved the dietary intakes of dark green leafy vegetables, legumes and discretionary foods within the intervention arm.

Trial registration: Australian New Zealand Clinical Trials Registry; ACTRN12618000527268; registered 10 April 2018.

\section{Background}

The global prevalence of age-related macular degeneration (AMD) is expected to reach approximately 300 million by the year 2040.[1] This chronic degenerative disease impairs central vision which is vital for everyday tasks such as reading and driving[1]. The functional disability associated with AMD increases the risk of mental health concerns such as depression and anxiety.[2] Despite the enormous personal burden, treatment options for AMD are limited to costly anti-vascular endothelial growth factor injections for people with the neovascular (wet) form of AMD, while the other form of late AMD (dry atrophic) is irreversible and untreatable[1]. Fortunately, recent research literature supports dietary modulation as a potentially effective preventative and management strategy for AMD.[3-5] People with AMD are therefore recommended to increase their consumption of dark green leafy vegetables, fish and low glycaemic index (GI) foods such as wholegrains and legumes[3-8]. 
Despite this, an evidence-practice gap has been identified between these dietary recommendations and those actually practiced by people with AMD.[6, 9-18] This is largely due to a lack of clear-cut guidelines for patients and/or practitioners to follow; patients not having sufficient information and/or misconceptions regarding diet and AMD; inadequate explanation and reinforcement by eye-care practitioners; and lack of dietitian referral and support.[6, 9-17, 19, 20]

There have been few translational efforts to address this evidence-practice gap. Promising findings were recently published following a non-randomised educational intervention trial in the United Kingdom.[21] In this study, intervention participants received verbal nutrition advice for their AMD from an eyecare professional accompanied by a take-home leaflet summarising the key messages around nutrition and AMD; control participants received usual care (off-the-shelf brochure).[21] As a result of this intervention, participants in this arm increased their egg intake, gained greater confidence about diet and AMD links and were more motivated to practice health-protective behaviours compared to participants in the control arm.[21]

Our study is a novel, parallel randomised controlled trial (RCT) that was conducted to assess the effectiveness of a telephone-delivered intervention to impart and disseminate evidence-based dietary advice for people with AMD. [22] A telehealth approach was considered to be appropriate for people with AMD, due to a strong association between older age AMD and its subsequent impact on mobility as a result of central vision loss.[1] Furthermore, telephone-delivered interventions are proven to have several advantages over traditional face-to-face models[23] and were previously shown to improve adherence to dietary recommendations[24, 25], diet quality[26] and selfefficacy[24] in both older adults and adults with chronic diseases. Therefore, the aims of this study are to:

1. present the 3-months post-intervention follow up results of the RCT and;

2. report on the feasibility and acceptability of this telehealth program for AMD patients.

\section{Methods}

\section{Baseline period}

A total of 155 participants were recruited into the study between June 2018 to July 2019. This number exceeds the proposed 140 participants needed to sufficiently achieve the primary outcome with $80 \%$ power as significant at the $5 \%$ level and allowing for $10 \%$ drop-out rate. Most participants were recruited within New South Wales $(n=$ 150, 96.7\%) of which 137 participants were recruited from three Sydney-based private eye clinics and 12 participants were recruited from our research database listing people who consented to be contacted about future studies by our research team. The remaining five participants were recruited across Australia following media advertisements.

Inclusion criteria for the study was a) a physician diagnosis of any form of AMD in either eye; and (b) age > 50 years old. The following were exclusion criteria: (a) lack of sufficient English fluency; (b) unwilling to participate in the 4-month intervention program; and (c) inability to provide informed consent. Participants were successfully recruited when written consent and the following baseline questionnaires were completed and returned: general baseline questionnaire including demographics, medical history and vision function; a 145-item food frequency questionnaire including information on supplement use to capture usual intake over 12-months; and a short dietary questionnaire (SDQ-AMD)[28] to capture actual intake of key food groups in the last seven days. Following recruitment, participants were randomised into one of two arms: intervention or control. The 
randomisation sequence was generated centrally using permuted blocks of mixed size to ensure a 1:1 allocation ratio while maintaining an unpredictable sequence. Assignments to the intervention or control arm was managed centrally so treating and recruiting staff were not involved in the process, however neither participants nor research staff could be blinded to the assignment allocations. All participants continued to receive usual care for their AMD by their eye-care professional.

This study was conducted in accordance to the Declaration of Helsinki. The protocol for the RCT has been published previously[22] and was approved by The University of Sydney Human Ethics Committee (Reference: HREC 2018/219). The CONSORT checklist (Additional File 1) and TIDiER checklist (Additional File 2) have been completed.

\section{Intervention period}

The intervention ran between August 2018-October 2019 and included a two-pronged approach: (1) develop and distribute a resource or workbook incorporating messages based on the latest Australian Dietary Guidelines[29] and evidence-based information on diet and AMD to the participant; and (2) have an accredited practising dietitian provide monthly telephone coaching and support for four months to facilitate and enhance the participants' adoption of AMD-specific dietary recommendations. The key dietary messages of this intervention included: i) increase consumption of dark green leafy vegetables, ii) eat fresh fruit daily, iii) choose low GI foods, iv) eat fish at least twice per week, and v) consume nuts two to three times per week.

The anticipated duration of each phone call was 20 minutes or a total of 80 minutes over four months. Any missed consultations for the month were logged by the dietitian to assess adherence at the end of the intervention. Number of call attempts and call duration were also logged. The intervention calls were tailored according to the '4 A's approach'[30]: 1) assessment (feedback) of participant diet and stage of change; 2) advice on optimal dietary behaviours; 3) assistance with collaborative goal setting; and 4) arranging follow up support i.e. next phone call.[22] The stages of change used in this study were categorised as: pre-contemplation (not thinking about change); contemplation (thinking about change within the next six months); preparation (making a change within the next month); action (currently trying to change); or maintenance (maintaining a change).[31]

The control arm received freely available off-the-shelf brochures about AMD and nutrition and were also briefly followed up by a member of the research team once per month during the intervention period. Phone call discussions included queries about the control package or study and/or any relevant comments and updates.

\section{Follow-up period}

Immediately following the 4-month intervention period, intervention participants were asked to complete a feedback form and repeat the SDQ-AMD that was administered at baseline. The feedback form was used to determine the acceptability of the intervention and included a 5-point Likert scale to assess satisfaction with the telehealth component and evidence-based resource; and two 'yes/no' questions: (1)'Would you feel confident in recommending this treatment to a friend?; and (2) 'Was it worth your time doing the program?'. A 5-point Likert scale was also used to determine self-assessed adherence to the intervention program, and two free response questions allowed participants to reflect on the most useful component of the intervention and provide additional feedback such as suggestions to improve the program. A second follow-up was conducted 3-months postintervention, inviting both intervention and control participants to repeat the SDQ-AMD.

\section{Outcome Measures}


As specified in the published protocol[22], the primary outcome measure for this study is a 0.5 serve per day increase in total vegetable intake; an achievable improvement according to data from an Australian populationbased intervention.[27] Appreciable improvements in the dietary intakes of dark green leafy vegetables, fruit, low GI foods, fish and nuts are the secondary outcomes at the 3-month post-intervention follow up period.

\section{Analysis}

Questionnaire data was entered into developed templates in REDCap (a secure web-based application for managing online databases). Data was exported as Excel spreadsheets for per-protocol statistical analysis using SAS version 9.4. Chi-square tests and Fisher's exact test were used to compare baseline study characteristics between the control and intervention arms and confirm that participants were randomly allocated. Descriptive statistics were used to describe the participants, call frequency, call duration, and dietary intakes. T-tests were used to compare dietary changes within and between each study arm at applicable follow-up periods with adjustments for age and sex. McNemar's test was used to compare the change in proportion of participants meeting the dietary goals. Level of significance for all statistical analyses was $p<0.05$.

\section{Results}

\section{Participants}

Figure 1 describes the trial profile where 155 participants were recruited at baseline (control, $n=78$; intervention, $n$ $=77$ ). The overall withdrawal rate at the 3-months post-intervention follow up was $5 \%(n=8 ; 3$ intervention, 5 control). Participant baseline characteristics are shown in Table 1 and differences in study characteristics between the study arms were mostly non-significant (exceptions for height and type of AMD). 
Table 1

Baseline characteristics of participants

\begin{tabular}{|llll|}
\hline Baseline Characteristics & Intervention $(\mathbf{n}=\mathbf{7 7})$ & Control $(\mathbf{n}=\mathbf{7 8})$ & P-value \\
\hline Age (years) & $78.1 \pm 8.1$ & $77.9 \pm 8.5$ & 0.88 \\
\hline Sex (\% female) & 50.7 & 64.1 & 0.09 \\
\hline Weight (kg) & $75.0 \pm 15.6$ & $70.7 \pm 14.0$ & 0.08 \\
\hline Height (cm) & $167.2 \pm 11.5$ & $161.5 \pm 9.5$ & 0.002 \\
\hline BMI (kg/m $\left.{ }^{2}\right)$ & $26.8 \pm 4.9$ & $27.0 \pm 5.4$ & 0.82 \\
\hline Type of AMD: & & & \\
No. eyes with early AMD & 6 & 0 & 0.01 \\
\hline No. eyes with any late AMD & 94 & 104 & \\
\hline Cardiovascular disease (n, \%) & $29.0(37.7)$ & $26.0(33.3)$ & 0.57 \\
\hline Stroke (n, \%) & $8.0(10.4)$ & $7.0(9.0 \%)$ & 0.77 \\
\hline High blood pressure (n, \%) & $49.0(63.6)$ & $49.0(62.8)$ & 0.92 \\
\hline High cholesterol (n, \%) & $41.0(53.3)$ & $38.0(50.7)$ & 0.75 \\
\hline Diabetes (n, \%) & $15.0(19.7)$ & $21.0(26.9)$ & 0.29 \\
\hline Kidney disease (n, \%) & $6.0(7.8)$ & $4.0(5.2)$ & 0.51 \\
\hline Physical activity (hrs/week) & $3.6 \pm 4.0$ & $4.4 \pm 7.7$ & 0.41 \\
\hline No. smokers (\%) & $34(44.7)$ & $28(35.9)$ & 0.35 \\
\hline Bolded values indicate statistical significance, p-value< 0.05 & \\
\hline
\end{tabular}

Within the intervention arm, the dietitian's self-assessed stage of change for each participant to make dietary modifications were: precontemplation $(26 \%, n=20)$, contemplation $(10 \%, n=8)$, preparation $(42 \%, n=32)$, action $(18 \%, n=14)$, and maintenance $(4 \%, n=3)$.

\section{Dietary intakes (expressed as mean serves \pm SE) between study arms}

The dietary intakes as assessed by the SDQ-AMD between study arms at baseline and at 3-months postintervention are shown in Table 2. Unadjusted baseline dietary intakes between intervention and control participants were not significantly different $(p>0.05)$ and specific $p$-values can be found in Additional File 3 . At the 3-months post-intervention follow up, adjusted mean intake of nuts was significantly higher in the intervention arm than the control arm ( $3.96 \pm 0.51$ vs $2.71 \pm 0.32, p=0.04)$. Unadjusted mean intakes at 3-months postintervention can be found in Additional File 4. 
Table 2

Age-sex adjusted mean dietary intakes at baseline and 3-months post-intervention

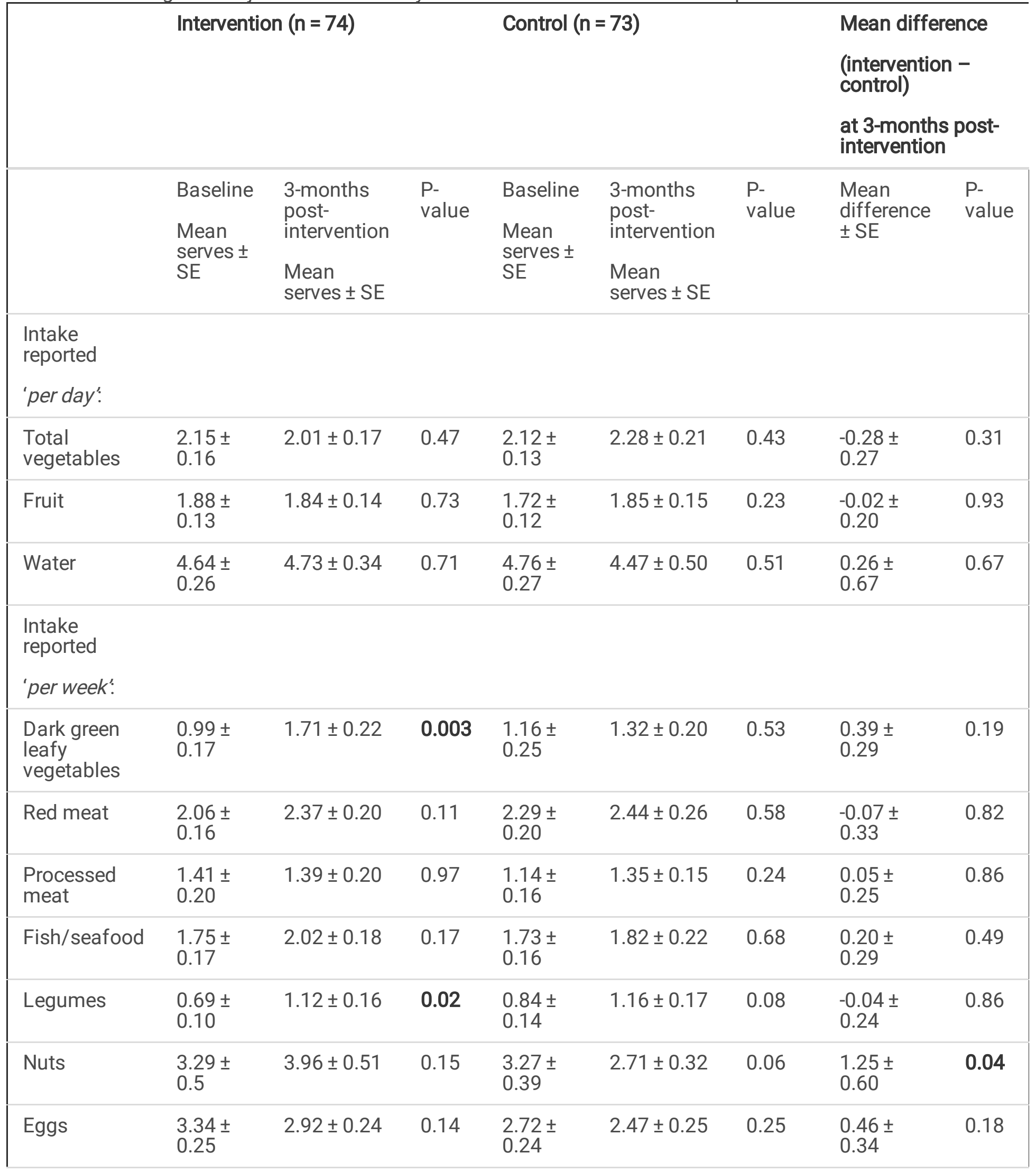

Bolded values indicate statistical significance, $p$-value $<0.05$. 


\begin{tabular}{|c|c|c|c|c|c|c|c|c|}
\hline \multirow{3}{*}{$\begin{array}{l}\text { Bread: } \\
\text { Wholemeal, } \\
\text { grain, rye, } \\
\text { sourdough }\end{array}$} & \multicolumn{3}{|c|}{ Intervention $(n=74)$} & \multicolumn{3}{|c|}{ Control $(n=73)$} & \multicolumn{2}{|c|}{$\begin{array}{l}\text { Mean difference } \\
\text { (intervention - } \\
\text { control) } \\
\text { at 3-months post- } \\
\text { intervention }\end{array}$} \\
\hline & $\begin{array}{l}5.06 \pm \\
0.50\end{array}$ & $4.71 \pm 0.48$ & 0.58 & \multirow{2}{*}{$\begin{array}{l}4.53 \pm \\
0.53 \\
1.63 \pm \\
0.31\end{array}$} & \multirow{2}{*}{$\begin{array}{l}4.26 \pm 0.63 \\
1.73 \pm 0.31\end{array}$} & \multirow{2}{*}{$\begin{array}{l}0.49 \\
0.72\end{array}$} & \multirow{3}{*}{$\begin{array}{l}0.46 \pm \\
0.63 \\
-0.41 \pm \\
0.43\end{array}$} & \multirow{3}{*}{$\begin{array}{l}0.47 \\
0.35\end{array}$} \\
\hline & $\begin{array}{l}1.41 \pm \\
0.30\end{array}$ & $1.33 \pm 0.30$ & 0.85 & & & & & \\
\hline White & & & & & & & & \\
\hline $\begin{array}{l}\text { Cakes, } \\
\text { biscuits, ice } \\
\text { cream, } \\
\text { processed } \\
\text { potato, } \\
\text { takeaway, } \\
\text { sugar } \\
\text { sweetened } \\
\text { beverages }\end{array}$ & $\begin{array}{l}8.31 \pm \\
0.76\end{array}$ & $6.54 \pm 0.58$ & 0.01 & $\begin{array}{l}8.82 \pm \\
0.82\end{array}$ & $6.84 \pm 0.62$ & 0.0003 & $\begin{array}{l}-0.30 \pm \\
0.85\end{array}$ & 0.73 \\
\hline Alcohol & $\begin{array}{l}3.72 \pm \\
0.83\end{array}$ & $2.93 \pm 0.53$ & 0.30 & $\begin{array}{l}2.18 \pm \\
0.48\end{array}$ & $2.58 \pm 0.51$ & 0.40 & $\begin{array}{l}0.35 \pm \\
0.74\end{array}$ & 0.64 \\
\hline Fats and oils: & $\begin{array}{l}2.14 \pm \\
0.28\end{array}$ & $2.05 \pm 0.28$ & 0.71 & $\begin{array}{l}2.58 \pm \\
0.30\end{array}$ & $2.59 \pm 0.31$ & 0.98 & $\begin{array}{l}-0.54 \pm \\
0.42\end{array}$ & 0.20 \\
\hline $\begin{array}{l}\text { Olive oil } \\
\text { Other }\end{array}$ & $\begin{array}{l}5.72 \pm \\
0.44\end{array}$ & $5.48 \pm 0.39$ & 0.59 & $\begin{array}{l}6.57 \pm \\
0.45\end{array}$ & $6.31 \pm 0.50$ & 0.57 & $\begin{array}{l}-0.83 \pm \\
0.63\end{array}$ & 0.19 \\
\hline
\end{tabular}

\section{Dietary intakes (expressed as mean serves \pm SE) within the RCT arms}

\section{Immediately post-intervention}

Within the intervention arm, there were significant improvements in dietary intake at the immediate postintervention follow up compared to baseline (Additional Table 5). This included significant improvements in daily water intake ( $5.36 \pm 0.27$ vs $4.63 \pm 0.26 ; p=0.01)$ and weekly intakes of: fish/seafood $(2.36 \pm 0.18$ vs $1.79 \pm 0.17$; $p=0.006)$, dark green leafy vegetables $(1.95 \pm 0.27$ vs $1.01 \pm 0.17 ; p=0.001)$, and eggs ( $3.78 \pm 0.29$ vs $3.32 \pm 0.25$;

$p=0.049$ ). Non-significant improvements in intakes of total vegetables, fruit, red and processed meats, legumes, nuts, specified discretionary food items, alcohol, and olive oil were also observed.

\section{3-months post-intervention}

The dietary intakes at the 3-months post-intervention follow up are shown in Table 2 and comparisons of these are presented for both arms. Participants who underwent the intervention had significantly higher age-sexadjusted mean intakes of dark green leafy vegetables $(1.71 \pm 0.22$ vs $0.99 \pm 0.17, p=0.003)$ and legumes $(1.12 \pm$ 
0.16 vs $0.69 \pm 0.10, p=0.02)$ at the 3 -month follow-up than baseline. Further, intakes of specified discretionary food items reduced significantly $(6.54 \pm 0.58$ vs $8.31 \pm 0.76, p=0.01)$ compared to baseline. The control arm also showed significantly reduced intakes of specified discretionary food items compared to baseline $(6.84 \pm 0.62$ vs $8.82 \pm 0.82, p=0.0002)$.

Figure 2 illustrates the proportion of participants at 3-months post-intervention from both arms meeting key dietary goals based on the Australian Dietary Guidelines[29] and recommendations presented in research literature for $\operatorname{AMD}[3,13,32-35]$. There were statistically significant increases in the proportion of intervention participants meeting the goals for: dark green leafy vegetables $(+18.9 \%, p=0.01)$, legumes $(+16.2 \%, p=0.02)$ and nuts $(+13.5 \%, p=0.03)$ compared to baseline (Additional File 6$)$. Within the control arm there was a significant increase in the proportion of participants meetings the recommendations for legumes $(+14 \%, p=$ 0.01). No significant differences were found between the study arms at 3-months post-intervention.

\section{Intervention adherence and acceptability}

During the 4-month intervention period, an average of $6.5(\mathrm{SD}=2.2)$ call attempts were made per intervention participant with $3.9(S D=0.6)$ successful calls. The total duration of calls per participant averaged 75.8 minutes $(S D=23.1$ minutes).

Most participants $(94.9 \%, n=74)$ completed the feedback form with almost all completers $(98.6 \%, n=73)$ being 'satisfied' or 'very satisfied' with both the evidence-based nutrition resource and telehealth calls; one participant was neutral to both. Similarly, $98.6 \%(n=73)$ of completers would recommend the intervention to others, and all participants who completed the feedback form thought the intervention was worth participating. Almost three quarters of the intervention participants $(72 \%, n=53)$ self-reported good to high adherence to the program. Some participants cited reduced oral intake secondary to poor appetite or health, and inflexible diets due to established menus at nursing homes/aged care facilities as reasons for poorer adherence.

Below are some participants comments about the intervention program. Participants are identified using individual identification numbers.

Most participants valued the monthly telephone calls which were tailored according to each participants' needs:

Simple explanations and good suggestions about food substitutes if different foods are not liked or tolerated (029)

Regular follow up which reinforced diet preference and benefits (033)

Talking to [the dietitian] when I was getting lazy cooking for myself nightly, encouraged me to go back to a healthy eating practice (142)

The phone calls review my previous month's diet. It made me think about what I had eaten and encouraged me to eat better in the next month (146)

The evidence-based resource was also valuable to participants to help remind and prompt them about healthy eating practices: 
I keep referring to the 'Dietary Recommendations for AMD' so that I'Il keep track (082)

It was more the reminder to keep on the healthy foods and what are the best to have (053)

I found the dietary sheets very helpful for planning the week meals and shopping (054)

A few participants reported having a healthy diet but appreciated the intervention to help reinforce their nutrition knowledge:

Confirmation of food choices. Recommendation of foods that were more beneficial (015)

Although I have a good diet it was useful to get guidance and keep up to date (108)

Helpful advice...found it reassuring as my diet has always included the foods recommended so now more focused on eating them regularly (130)

\section{Discussion}

This is the first study to evaluate an evidence-based telehealth intervention program designed specifically to improve the dietary intakes and behaviours of people with AMD. Overall, the intervention was delivered as planned for most participants with a total of 3.9 out of the intended four successful phone calls made per participant and an average total call duration of 75 minutes compared to the expected 80 minutes over the four months. The program was also well-received and appreciated by the participants and led to statistically significant and clinically meaningful improvements in dietary intakes over a 3-month follow-up.

Some of the key dietary recommendations for AMD published in the literature are to include: at least two serves per week of fish/seafood which is associated with reduced risk of early and neovascular AMD $[3-5,13,36]$; at least two serves per week of dark green leafy vegetables which is recommended to obtain the benefits of dietary carotenoids[3]; and two to four eggs per week which has been linked to reduced risk of AMD progression, with eggs additionally being a bioavailable source of lutein and zeaxanthin[5, 37]. In our study, the intervention arm significantly increased their mean intakes to meet these particular recommendations immediately after the intervention. These improvements may be due to the program's emphasis on AMD-specific dietary advice rather than general healthy eating advice such as increasing total vegetables and fruit intake.

At the 3-months post-intervention follow up, intakes of dark green leafy vegetables continued to be significantly higher compared to baseline intakes suggesting that this particular dietary modification may have been more feasible to incorporate and maintain in the diet than the other recommendations. However, mean fish/seafood intake at 3-months post-intervention continued to meet the recommended $\geq$ two serves per week[3] and was approximately 0.3 serves higher than baseline. This difference is equivalent to a $30 \mathrm{~g}$ increase over the week, where a serve of fish/seafood is $100 \mathrm{~g}$ according to the Australian Dietary Guidelines[29]. Although, we did not find this result to be statistically significant, research literature suggests that this increase has clinical importance for general health as a multi-cohort cross-cultural study investigating the diets of older adults in Australia, Greece, Japan and Sweden, reported that every $20 \mathrm{~g}$ increase in fish and shellfish intake was significantly associated with a $6 \%$ reduction in hazard of death, after accounting for ethnicity.[38] Comparatively, our control arm's mean 
fish/seafood intake increased by approximately $10 \mathrm{~g}$ ( 0.1 serve) to 1.82 serves per week which does not have statistical significance and less likely to be of clinical significance. This suggests that more targeted interventions than standard brochures may be needed to change dietary behaviour.

The study by Darmadi-Blackberry et al. also reported that every $20 \mathrm{~g}$ increase in legume intake was significantly associated with an $8 \%$ reduction in risk of death, irrespective of ethnicity.[38] In our study, both the intervention and control arms increased their mean legume intake by more than $20 \mathrm{~g}$ (where a serve of legumes is 150g[29]) at the 3-months post-intervention follow-up compared to baseline. However, the increase within the intervention arm was $25 \%$ higher than the control arm further supporting the effectiveness of the program.

In addition to improvements within the intervention arm, the program appears to have led to a difference in dietary intakes between study arms at 3-months post-intervention. The most notable difference being a significantly higher mean intake of nuts in the intervention arm (four serves per week) compared to the control arm ( 2.7 serves per week). The intervention arm also achieved better intakes of other food items compared to the control arm, however, these were non-significant. Interestingly, mean intakes of total vegetables and fruit were nonsignificantly higher in the control arm than the intervention arm at this follow up. Possible reasons for this difference could again be due to the program's focus on AMD-specific recommendations rather than general healthy eating advice that was provided to the control arm or might be due to individual preferences for seasonal produce.

Overall, the positive dietary changes reported in this study may be the result of a culmination of factors. A study evaluating nutrition interventions amongst older adults reported that dietary modification was more successful in studies that included participants with a specific health condition.[39] This may be due to increased motivation to manage the condition as most (60\%) of our intervention participants were in the 'preparation' or 'action' stage of change at the start of the program. Furthermore, collaborative goal setting and regular contact with a health professional, which are incorporated within the 4 A's approach of our intervention[30], have been shown to be successful nutrition education intervention components leading to better behavioral outcomes in older adults.[30, 39 , 40] However, more significant dietary improvements may have been observed if our program limited its focus to one or two messages[39] such as increasing intakes of dark green leafy vegetables and fish/seafood rather than also including messages around other food groups/items like nuts and legumes.

\section{Strengths and Limitations}

There are several strengths to this study. Firstly, this is a novel program designed to specifically improve the dietary intakes and behaviours of people with AMD and was tested using the "gold standard" RCT study design. Secondly, this program involved a collaborative effort between accredited practising dietitians and experts in the field of AMD (retinal specialists and epidemiologists) to provide evidence-based care. Thirdly, this program incorporates a telehealth component which is particularly suited to study participants who were typically older adults with functional limitations (vision and mobility) and other pre-existing health problems. Acceptance of a telehealth program is especially relevant in light of the current coronavirus disease-19 (COVID-19) pandemic which has emphasised the importance of telehealth services to provide safe and accessible healthcare to vulnerable subsets of the population.[41] Our study findings therefore reinforce the advantages of telehealth programs and confirm that safe and effective dietary advice and counselling can be provided to older adults in this format. 
However, no study is without limitations and we acknowledge that the results reported in this study may not reflect usual intake as the SDQ-AMD tool collects actual intake in the last week of a limited number of food items. In addition, this tool does not include a question about supplements use and therefore we are unable to report on participants' use of the recommended Age-Related Eye Disease Study (AREDS) supplements for AMD. To overcome these limitations, the food frequency questionnaire, which collects usual dietary intake of 145 items and supplement use in the last 12 months, is currently being re-administered alongside the SDQ-AMD at the final follow-up (i.e. 6-months post-intervention). This data will then be compared with baseline data to provide further valuable insight into the usual dietary intakes and behaviour changes of patients with AMD.

\section{Conclusion}

This telehealth program appears to have effectively improved the dietary intakes of people with AMD. At 3months post-intervention, there were significant increases in intakes of dark green leafy vegetables and legumes within the intervention arm, and significantly higher intakes of nuts than the control arm.

\section{Abbreviations}

AMD: Age-related macular degeneration

AREDS: Age-related eye disease study

GI: Glycaemic index

SDQ-AMD: Short dietary questionnaire - age-related macular degeneration

\section{Declarations}

\section{Ethics approval and consent to participate:}

The protocol for this study was approved by The University of Sydney Human Ethics Committee (Reference: HREC 2018/219). All participants provided written informed consent prior to entering the study.

\section{Consent for publication:}

Not applicable.

\section{Availability of data and materials:}

The datasets used and/or analysed during the current study are available from the corresponding author on reasonable request. The study protocol has been published.

\section{Competing interests:}

None declared.

\section{Funding:}

This work is supported by NHMRC grant number APP1150101. 


\section{Authors' contributions:}

BG obtained funding for the research. DT, BG, VF, PM, GL contributed towards the study design and concept. DT was involved in data collection. DT and GB were involved in data analysis and interpretation. DT drafted the manuscript with critical input from all other authors. The final version was approved by all authors.

\section{References}

1. Mitchell P, Liew G, Gopinath B, Wong TY. Age-related macular degeneration. The Lancet 2018;392:1147-59. https://doi.org/10.1016/S0140-6736(18)31550-2.

2. Cimarolli VR, Casten RJ, Rovner BW, Heyl V, Sörensen S, Horowitz A. Anxiety and depression in patients with advanced macular degeneration: Current perspectives. Clinical Ophthalmology 2016;10:55-63. https://doi.org/10.2147/OPTH.S80489.

3. Broadhead GK, Grigg JR, Chang AA, McCluskey P. Dietary modification and supplementation for the treatment of age-related macular degeneration. Nutr Rev 2015;73:448-62. https://doi.org/10.1093/nutrit/nuv005.

4. Tan JSL, Wang JJ, Flood V, Rochtchina E, Smith W, Mitchell P. Dietary Antioxidants and the Long-term Incidence of Age-Related Macular Degeneration. The Blue Mountains Eye Study. Ophthalmology 2008;115:334-41. https://doi.org/10.1016/j.ophtha.2007.03.083.

5. Eisenhauer B, Natoli S, Liew G, Flood VM. Lutein and Zeaxanthin-Food Sources, Bioavailability and Dietary Variety in Age-Related Macular Degeneration Protection. Nutrients 2017;9. https://doi.org/10.3390/nu9020120.

6. Wang JJ, Rochtchina E, Smith W, Klein R, Klein BEK, Joshi T, et al. Combined Effects of Complement Factor H Genotypes, Fish Consumption, and Inflammatory Markers on Long-Term Risk for Age-related Macular Degeneration in a Cohort. American Journal of Epidemiology 2009;169:633-41. https://doi.org/10.1093/aje/kwn358.

7. Chew EY, Clemons TE, Sangiovanni JP, Danis RP, Ferris 3rd FL, Elman MJ, et al. Secondary analyses of the effects of lutein/zeaxanthin on age-related macular degeneration progression: AREDS2 report No. 3. JAMA Ophthalmol 2014;132:142-9. https://doi.org/10.1001/jamaophthalmol.2013.7376.

8. Chew EY, Clemons TE, SanGiovanni JP, Danis R, Ferris FL, Elman M, et al. Lutein + zeaxanthin and omega-3 fatty acids for age-related macular degeneration: The Age-Related Eye Disease Study 2 (AREDS2) randomized clinical trial. JAMA - Journal of the American Medical Association 2013;309:2005-15. https://doi.org/10.1001/jama.2013.4997.

9. Shah SU, Pilli S, Telander DG, Morse LS, Park SS. Survey of patients with age-related macular degeneration: knowledge and adherence to recommendations. Can J Ophthalmol 2013;48:204-9. https://doi.org/10.1016/j.jcjo.2013.01.013.

10. Nunes S, Alves D, Barreto P, Raimundo M, da Luz Cachulo M, Farinha C, et al. Adherence to a Mediterranean diet and its association with age-related macular degeneration. The Coimbra Eye Study-Report 4. Nutrition 2018;51-52:6-12. https://doi.org/10.1016/j.nut.2017.12.010.

11. Kim EK, Kim H, Kwon O, Chang N. Associations between fruits, vegetables, vitamin A, beta-carotene and flavonol dietary intake, and age-related macular degeneration in elderly women in Korea: the Fifth Korea 
National Health and Nutrition Examination Survey. Eur J Clin Nutr 2018;72:161-7.

https://doi.org/10.1038/ejcn.2017.152.

12. Cho E, Hung S, Willett W, Spiegelman D, Rimm E, Seddon J, et al. Prospective study of dietary fat and the risk of age-related macular degeneration. Am J Clin Nutr 2001;73:209-18.

https://doi.org/10.1093/ajcn/73.2.209.

13. SanGiovanni JP, Chew EY, Clemons TE, Davis MD, Ferris 3rd FL, Gensler GR, et al. The relationship of dietary lipid intake and age-related macular degeneration in a case-control study: AREDS Report No. 20. Arch Ophthalmol 2007;125:671-9. https://doi.org/10.1001/archopht.125.5.671.

14. Amirul Islam FM, Chong EW, Hodge AM, Guymer RH, Aung KZ, Makeyeva GA, et al. Dietary patterns and their associations with age-related macular degeneration: the Melbourne collaborative cohort study.

Ophthalmology 2014;121:1428-1434.e2. https://doi.org/10.1016/j.ophtha.2014.01.002.

15. Merle BMJ, Silver RE, Rosner B, Seddon JM. Adherence to a Mediterranean diet, genetic susceptibility, and progression to advanced macular degeneration: A prospective cohort study. American Journal of Clinical Nutrition 2015;102:1196-206. https://doi.org/10.3945/ajcn.115.111047.

16. Hogg RE, Woodside J v, McGrath A, Young IS, Vioque JL, Chakravarthy U, et al. Mediterranean Diet Score and Its Association with Age-Related Macular Degeneration: The European Eye Study. Ophthalmology 2017;124:82-9. https://doi.org/10.1016/j.ophtha.2016.09.019.

17. Gopinath B, Flood VM, Kifley A, Liew G, Mitchell P. Smoking, Antioxidant Supplementation and Dietary Intakes among Older Adults with Age-Related Macular Degeneration over 10 Years. PLOS ONE 2015;10:e0122548. https://doi.org/10.1371/journal.pone.0122548.

18. National Institute for Health and Care Excellence (NICE). Age-related macular degeneration: NICE guideline 2018. https://www.nice.org.uk/guidance/ng82/chapter/Recommendations\#information-and-support (accessed June 24, 2020).

19. Ng WT, Goggin M. Awareness of and compliance with recommended dietary supplement among age-related macular degeneration patients. Clin Exp Ophthalmol 2006;34:9-14. https://doi.org/10.1111/j.14429071.2006.01141.x.

20. Lawrenson JG, Evans JR, Downie LE. A critical appraisal of national and international clinical practice guidelines reporting nutritional recommendations for age-related macular degeneration: Are recommendations evidence-based? Nutrients 2019;11. https://doi.org/10.3390/nu11040823.

21. Stevens $\mathrm{R}$, Cooke $\mathrm{R}$, Bartlett $\mathrm{H}$. Testing the impact of an educational intervention designed to promote ocular health among people with age-related macular degeneration. British Journal of Visual Impairment 2018;36:110-27. https://doi.org/10.1177/0264619617735144.

22. Tang D, Mitchell P, Flood V, Kifley A, Hayes A, Liew G, et al. Dietary intervention in patients with age-related macular degeneration: protocol for a randomised controlled trial. BMJ Open 2019;9:e024774-e024774. https://doi.org/10.1136/bmjopen-2018-024774.

23. Goode AD, Reeves MM, Eakin EG. Telephone-delivered interventions for physical activity and dietary behavior change: An updated systematic review. American Journal of Preventive Medicine 2012;42:81-8. https://doi.org/10.1016/j.amepre.2011.08.025.

24. van den Berg N, Schumann M, Kraft K, Hoffmann W. Telemedicine and telecare for older patients - A systematic review. Maturitas 2012;73:94-114. https://doi.org/10.1016/j.maturitas.2012.06.010. 
25. Kelly JT, Reidlinger DP, Hoffmann TC, Campbell KL. Telehealth methods to deliver dietary interventions in adults with chronic disease: A systematic review and meta-analysis1,2. American Journal of Clinical Nutrition 2016;104:1693-702. https://doi.org/10.3945/ajcn.116.136333.

26. Desroches S, Lapointe A, Ratté S, Gravel K, Légaré F, Turcotte S. Interventions to enhance adherence to dietary advice for preventing and managing chronic diseases in adults. Cochrane Database of Systematic Reviews 2013;2013. https://doi.org/10.1002/14651858.CD008722.pub2.

27. Pollard CM, Miller MR, Daly AM, Crouchley KE, O’Donoghue KJ, Lang AJ, et al. Increasing fruit and vegetable consumption: success of the Western Australian Go for $2 \& 5$ campaign. Public Health Nutr 2008;11:314-20. https://doi.org/10.1017/s1368980007000523.

28. Tang D, Mitchell P, Liew G, Burlutsky G, Flood V, Gopinath B. Evaluation of a Novel Tool for Screening Inadequate Food Intake in Age-Related Macular Degeneration Patients. Nutrients 2019;11:3031. https://doi.org/10.3390/nu11123031.

29. National Health and Medical Research Council. Eat for Health Australian Dietary Guidelines. Canberra, Australia: Canberra, Australia; 2013.

30. Goldstein MG, Whitlock EP, DePue J. Multiple behavioral risk factor interventions in primary care. Summary of research evidence. Am J Prev Med 2004;27:61-79. https://doi.org/10.1016/j.amepre.2004.04.023.

31. Campbell MK, DeVellis BM, Strecher VJ, Ammerman AS, DeVellis RF, Sandler RS. Improving dietary behavior: the effectiveness of tailored messages in primary care settings. American Journal of Public Health 1994;84:783-7. https://doi.org/10.2105/ajph.84.5.783.

32. Chiu CJ, Klein R, Milton RC, Gensler G, Taylor A. Does eating particular diets alter the risk of age-related macular degeneration in users of the Age-Related Eye Disease Study supplements? Br J Ophthalmol 2009;93:1241-6. https://doi.org/10.1136/bjo.2008.143412.

33. Kaushik S, Wang JJ, Flood V, Tan JS, Barclay AW, Wong TY, et al. Dietary glycemic index and the risk of agerelated macular degeneration. Am J Clin Nutr 2008;88:1104-10. https://doi.org/10.1093/ajcn/88.4.1104.

34. Chiu C-J, Milton RC, Klein R, Gensler G, Taylor A. Dietary carbohydrate and the progression of age-related macular degeneration: a prospective study from the Age-Related Eye Disease Study. Am J Clin Nutr 2007;86:1210-8. https://doi.org/10.1093/ajcn/86.4.1210.

35. Grains and Legumes Nutrition Council. Lifting the lid on legumes: the benefits of choosing legumes 2013. https://www.glnc.org.au/wp-content/uploads/2011/04/GLN_LiftingtheLid_WEB.pdf (accessed October 29, 2019).

36. Tan JSL, Wang JJ, Flood V, Mitchell P. Dietary fatty acids and the 10-year incidence of age-related macular degeneration: The blue mountains eye study. Archives of Ophthalmology 2009;127:656-65. https://doi.org/10.1001/archophthalmol.2009.76.

37. Gopinath B, Liew G, Tang D, Burlutsky G, Flood VM, Mitchell P. Consumption of eggs and the 15-year incidence of age-related macular degeneration. Clin Nutr 2019. https://doi.org/10.1016/j.clnu.2019.03.009.

38. Darmadi-Blackberry I, Wahlqvist ML, Kouris-Blazos A, Steen B, Lukito W, Horie Y, et al. Legumes: the most important dietary predictor of survival in older people of different ethnicities - PubMed. Asia Pacific Journal Clinical Nutrition 2004;13:217-20.

39. Sahyoun NR, Pratt CA, Anderson A. Evaluation of nutrition education interventions for older adults: A proposed framework. Journal of the American Dietetic Association 2004;104:58-69. https://doi.org/10.1016/j.jada.2003.10.013. 
40. Bandayrel K, Wong S. Systematic Literature Review of Randomized Control Trials Assessing the Effectiveness of Nutrition Interventions in Community-Dwelling Older Adults. Journal of Nutrition Education and Behavior 2011;43:251-62. https://doi.org/10.1016/j.jneb.2010.01.004.

41. Wosik J, Fudim M, Cameron B, Gellad ZF, Cho A, Phinney D, et al. Telehealth transformation: COVID-19 and the rise of virtual care | Journal of the American Medical Informatics Association | Oxford Academic. A Scholarly Journal of Informatics in Health and Biomedicine 2020;27:957-62.

\section{Figures}

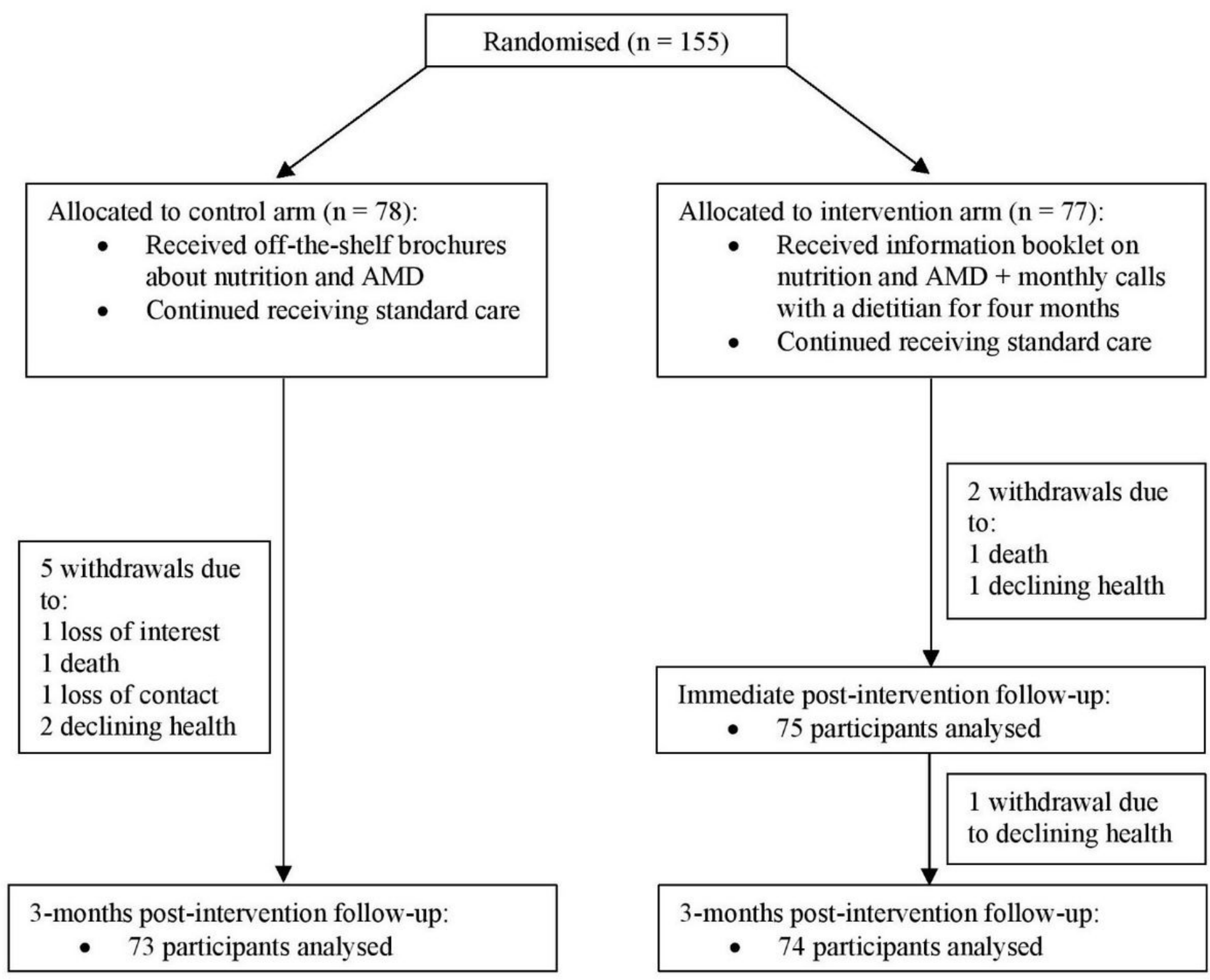

Figure 1

Trial profile 


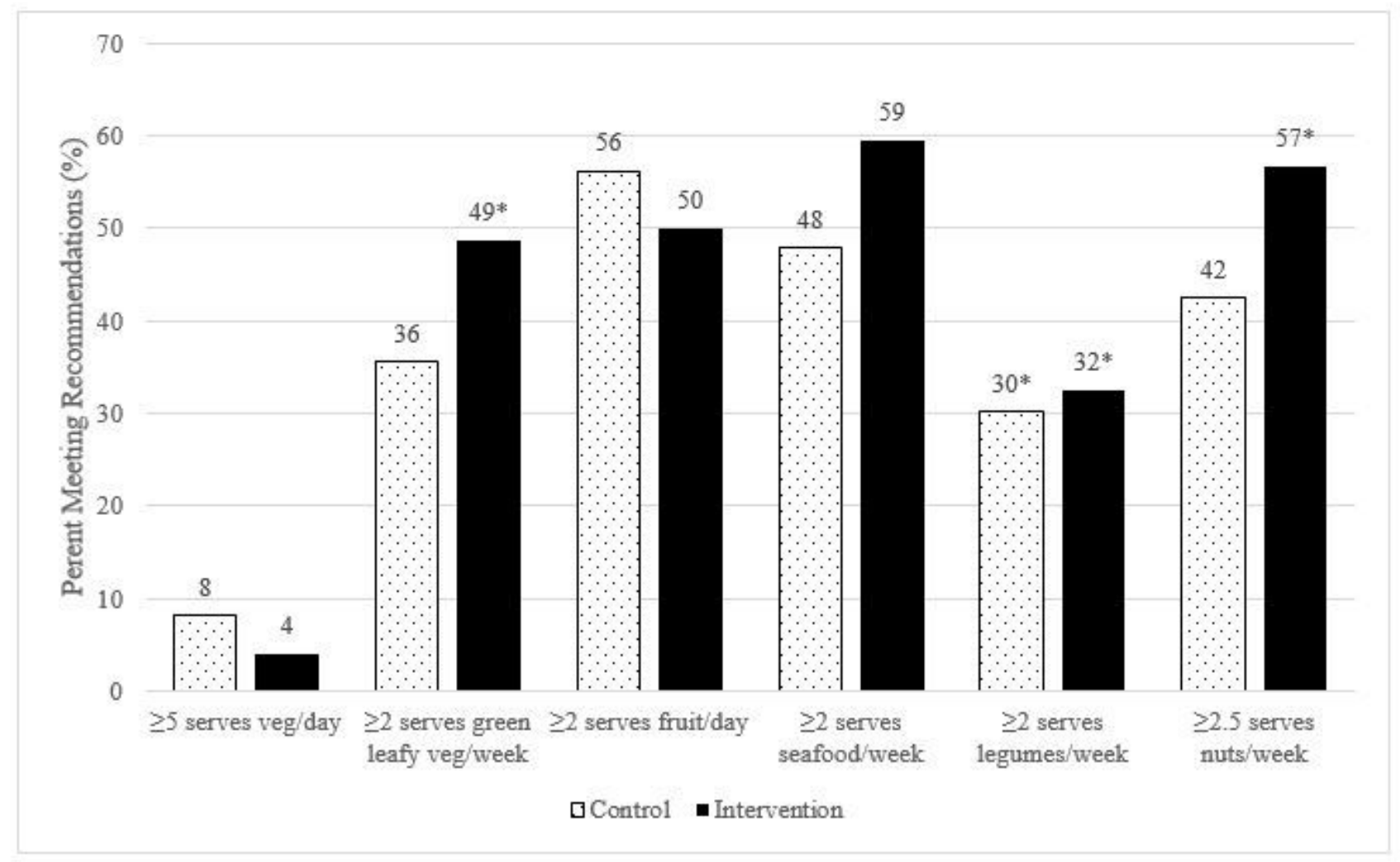

Figure 2

Proportion of participants meeting dietary goals at 3-months post-intervention. *Indicates a statistically significant $(p<0.05)$ increase within the study arm of the proportion of participants meeting the required intake compared to baseline. No significant difference in proportion was found between the study arms.

\section{Supplementary Files}

This is a list of supplementary files associated with this preprint. Click to download.

- AdditionalFile6.docx

- AdditionalFile5.docx

- AdditionalFile4.docx

- AdditionalFile3.docx

- AdditionalFile2.docx

- AdditionalFile1.doc 